\title{
The impact of heterogeneity on indeterminacy
}

Christian Ghiglino

Marielle Olszak-Duquenne

02-13

November 2002

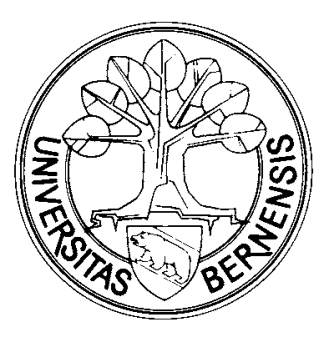

Universität Bern

Volkswirtschaftliches Institut

Gesellschaftstrasse 49

3012 Bern, Switzerland

Tel: 41 (0)31 6314506

Web: www-vwi.unibe.ch 


\title{
The impact of heterogeneity on indeterminacy
}

\author{
Christian Ghiglino $^{1 *}$ and Marielle Olszak-Duquenne ${ }^{2}$ \\ ${ }^{1}$ Institute of Economics, University of Bern, Gesellschaftsstrasse 49, 3012 Bern, Switzer- \\ land. e-mail: chiristian.ghiglino@vwi.unibe.ch \\ and on leave from \\ Department of Economics, Queen Mary, University of London, Mile End Road, London \\ E1 4 NS UK \\ ${ }^{2}$ Departement d'Econometrie, University of Geneva, 102, Bd. Carl-Vogt, CH-1211 Geneve- \\ 4, Suisse
}

Summary. The aim of the paper is to explore the link between agent's heterogeneity and indeterminacy in a general equilibrium economy. The framework is provided by the two-sector growth model with technological externalities of Boldrin and Rustichini (1994) in which heterogeneous agents are introduced. We first show that the occurrence of indeterminacy depends on the distribution in labor endowments and in shares on initial capital among the agents as well as on preferences and technology. We find that the sign of the effect of heterogeneity on indeterminacy is not pinned down by the standard properties of preferences, a fact that might be surprising in view of some recent results (as in Herrendorf et al. (2000)). However, when risk aversion is a concave or a slightly convex function, then heterogeneity is a factor that opposes the external effects in generating indeterminacy.

Endogenous growth, Heterogeneity, Indeterminacy, Inequalities.

\section{JEL-classification numbers: D30, D50, D90, O41.}

*Corresponding author. On leave from the Department of Economics, Queen Mary, University of London, Mile End Road, London E1 4NS UK. 


\section{Introduction}

Recently, there have been claims that the occurrence of indeterminacy in models with externalities "is overstated in representative agent models, as these ignore the potential stabilizing effect of heterogeneity" (Herrendorf et al (2000). This remark may seem surprising from a standard general equilibrium viewpoint, as heterogeneity is usually a source of complexity because it provides many additional degrees of freedom to the economy. On the other hand, it is also well known that aggregation of heterogeneous agents may produce under certain circumstances an aggregate demand possessing some definite properties (e.g., Grandmont (1992)). The aim of the present paper is to explore the link between heterogeneity and indeterminacy in a general equilibrium model.

In the paper we show that heterogeneity may both favor or be opposed to indeterminacy in economies with external effects. However, under some plausible conditions on risk aversion, heterogeneity is a factor that tends to neutralize the externality as a source of indeterminacy. The results are obtained within the framework of a general equilibrium model of the type used by Bewley (1982) but with technological externalities. In order to investigate the dynamic properties of the model, the technology is specified analytically while preferences are kept general. In fact, the model we consider is similar to Boldrin and Rustichini (1994). The major innovation is that we abandon the representative agent assumption and admit non-linear utility functions. Agents may be heterogeneous in respect to the share of the initial stock of capital and in labor endowments as well as in preferences. Due to the structure of the model, individual characteristics and heterogeneity do not affect the steady state itself as far as aggregate variables are considered. However, this model is sufficient to analyze the effects of heterogeneity on indeterminacy. Note that the introduction of externalities in a model with heterogenous agents presents some well known technical difficulties (see Kehoe, Levine and Romer (1990), Santos (1992) or Ghiglino (2002)).

Heterogeneity has an effect on indeterminacy. However, the usual axioms on preferences do not limit the sign of this effect. The reason is that this depends on the third and higher order derivatives of the utility functions. Empirical data concerning these derivatives is lacking. However, risk aversion is linked to the curvature of the utility function and therefore to the advent of an indeterminate steady state. Data seems to indicate that risk aversion is, at least, slightly decreasing. A condition on the first order derivative of risk aversion is not enough to restrict the sign of the impact of heterogeneity on indeterminacy, while its concavity or slight convexity are sufficient conditions ensuring that heterogeneity is opposed to indeterminacy.

The tractability of the model is based on some strong simplifying assumptions. First, there is only one consumption good and one capital good. Second, technology is restricted to belong to some class as the production functions are analytically specified. Finally, labor endowments are considered as exogenous parameters. The model may be used to describe a variety of situations in which labor is provided inelastically. The first situation we 
have in mind is one in which the differences in labor endowments model taxes on current income that are included in a redistribution policy. However, the present model may also be used to gain intuition on the behavior of a more complex model in which labor supply is endogenously determined. In this case differences in labor endowments are a shortcut to model the differences in the disutilities that agents associate to labor. Heterogeneity in labor could also reflect differences in the levels of productivity across agents.

To our knowledge, this paper is the first attempt to analyze the link between indeterminacy and the redistribution of capital shares and labor endowments in a general equilibrium model with external effects. The scope of the present paper is similar to Herrendorf et al. (2000) as both papers analyze the impact of heterogeneity on indeterminacy. However, the two frameworks are very different as Herrendorf et al. consider a continuous time overlapping generations economy with exogenous prices (as in Matsuyama (1991), an assumption that eliminates most of the the general equilibrium effects. Finally, in that model agents differ in respect to their productivity. We adopt a general equilibrium framework with fully flexible prices. Agents are infinitely lived. Heterogeneity is in terms of the agents characteristics which are shares in initial capital, endowments in labor and preferences. As a special case we will focus on heterogeneity in shares only. The present paper is also related to Ghiglino and Sorger (2002). In that paper, indeterminacy is shown to occur in a continuous time, endogenous growth model with externalities and heterogeneous agents. However, their analysis fail to qualify the effects of redistributions on the occurrence of indeterminacy. Another weakness is that preferences are bound to be loglinear. A version without externalities of the present model is also considered by Ghiglino and Olszak-Duquenne (2001). In that paper it is shown that with no externalities the distribution of labor endowments and capital shares matters in the stability properties of the steady state. In the present paper we focus instead on the link between indeterminacy and redistribution and highlight the role played by risk aversion.

The paper is organized as follows: In section 2 the model is introduced while the equilibria are defined in Section 3. Section 4 focuses on the dynamics followed by the aggregate variables near the steady state and local indeterminacy. In section 5 the occurrence of indeterminacy in the model with heterogenous agents is analyzed. Finally, in Section 6 some sufficient conditions ensuring that heterogeneity reduces indeterminacy are obtained.

\section{The model}

In the present paper we consider a competitive two-sector economy with different types of agents and technology externalities. Since we focus on dynamics, the model need to be kept as tractable as possible. The technology is formalized as in Boldrin and Rustichini (1994) but we introduce heterogeneity across agents. The externalities are 
of the labor-augmenting type as detailed below. There is no joint-production and firms produce according to constant returns production functions so that at the optimum, profits are zero. There are two produced goods, a consumption good and a capital good. The consumption good cannot be used as capital so it is entirely consumed. The capital good cannot be consumed. There are two inputs, capital and labor. We also suppose that there is instantaneous capital depreciation and that labor is inelastically used in production. There are two firms, one for each sector. The firm in the first sector produces a consumption good with two inputs, capital and labor, according to a production function that include externalities from capital, $\hat{F}^{1}\left(k^{1}, \tilde{l}^{1}, k\right)$. The externality is assumed to be a labor-augmenting technological progress, i.e. $\hat{F}^{1}\left(k^{1}, \tilde{l}^{1}, k\right)=F^{1}\left(k^{1}, k^{\eta} \tilde{l}^{1}\right)$. Let $l^{1}=k^{\eta} \tilde{l}^{1}$ be the "effective" labor force and assume that $F^{1}$ is a Cobb-Douglas production function then $F^{1}\left(k^{1}, l^{1}\right)=\left(l^{1}\right)^{\alpha}\left(k^{1}\right)^{1-\alpha}$ with $\alpha \in(0,1)$ where $l^{1}, k^{1}$ are the amount of capital and "effective" labor used by the firm of the consumption sector.

In a decentralized economy, the firm maximizes profit

$$
\operatorname{Max} p_{t}^{1} F^{1}\left(k_{t-1}^{1}, l_{t}^{1}\right)-p_{t-1}^{2} k_{t-1}^{1}-w_{t} l_{t}^{1}
$$

where $p_{t}^{1}$ is the price of the consumption good at period $t, p_{t-1}^{2}$ is the price of the capital good at period $t-1$ and $w_{t}$ the price of labor at period $t$. The optimal production plan satisfies the first order conditions

$$
\begin{aligned}
& p_{t}^{1} \frac{\partial F^{1}}{\partial k}=p_{t-1}^{2} \\
& p_{t}^{1} \frac{\partial F^{1}}{\partial l}=w_{t}
\end{aligned}
$$

In the second sector, the externality is also a labor-augmenting technological progress so it can be treated as above. The representative firm produces a capital good according to a Leontief function $F^{2}\left(k^{2}, l^{2}\right)=\operatorname{Min}\left(l^{2}, \frac{k^{2}}{\gamma}\right)$ with $\gamma \in(0,1)$. The optimal production plan for this firm is then

$$
l_{t}^{2}=\frac{k_{t-1}^{2}}{\gamma}
$$

There are three types of agents, $a, b$ and $c$. There are $N_{i}$ agents of type $i, i=a, b, c$. The framework could be extended as to include any finite number of types. In each period consumers provide inelastically a constant amount of labor $\omega_{i}, i=a, b, c$ with $N_{a} \omega_{a}+N_{b} \omega_{b}+N_{c} \omega_{c}=1$. A model in which the amount of labor provided is endogenously determined could be analyzed but at a much higher cost. At the beginning of the economy, each agent $i$ is endowed with a fixed share $\theta_{i}$ of the initial stock $k_{-1}$ of capital, with $N_{a} \theta_{a}+N_{b} \theta_{b}+N_{c} \theta_{c}=1$. Consumer's preferences are characterized by a discounted utility function of the form

$$
U^{i}\left(x^{i}\right)=\sum_{t=0}^{\infty} \delta^{t} u_{i}\left(x_{i t}\right)
$$

where $x_{i t}$ is the consumption of agent $i$ at time $t$ and $x^{i}$ is its intertemporal consumption stream. We assume $\delta>\gamma$. The instantaneous utility function fulfills the Inada condition

$$
\lim _{x_{i t} \rightarrow 0} u^{\prime}\left(x_{i t}\right)=+\infty \text {. }
$$


In a decentralized economy, an agent $i$ maximizes his utility function subject to a single budget constraint

$$
\sum_{t=0}^{\infty} p_{t}^{1} x_{i t}=\sum_{t=0}^{\infty} w_{t} \omega_{i}+p_{-1}^{2} \theta_{i} k_{-1} \quad \text { with } i=a, b, c .
$$

\section{$3 \quad$ Equilibria and steady states}

In the present economy the first welfare theorem does not necessary hold. However, as was recognized by Kehoe, Levine and Romer (1992), every competitive equilibrium obtained in a decentralized economy is a Pseudo-Pareto optimum in the sense that is the solution to the maximization of a social welfare function (see Ghiglino (2002) for some applications of this approach). This function could be considered as the objective of a constrained central planner. In the current section we first define competitive equilibria and then characterize the set of Pseudo-Pareto optima.

\subsection{The competitive equilibrium}

A competitive equilibrium can be defined as a sequence satisfying the following definition. Note that, due to the form of the externality the total "effective" labor at time $t$ is the product of the work force with $k_{t-1}^{\eta}$.

Definition 1 A competitive equilibrium is a sequence of prices $\left(p_{t}^{1}, p_{t}^{2}, w_{t}\right)_{t=0}^{\infty}$ with $p_{-1}^{2}=1$ such that markets clear for every $t \geq 0$ :

- $l_{t}^{1}+l_{t}^{2}=\left(N_{a} \omega_{a}+N_{b} \omega_{b}+N_{c} \omega_{c}\right) k_{t-1}^{\eta}=k_{t-1}^{\eta}$

- $k_{t}^{1}+k_{t}^{2}=F^{2}\left(k_{t-1}^{2}, l_{t}^{2}\right)$

- $N_{a} x_{a t}+N_{b} x_{b t}+N_{c} x_{c t}=F^{1}\left(k_{t-1}^{1}, l_{t}^{1}\right)$

- $k_{-1}^{1}+k_{-1}^{2}=k_{-1}$ with $k_{-1}$ given

where

- $\left(x_{i t}\right)$ is a solution to the individual maximization program of agent $i, i=a, b, c$ for $\left(p_{t}^{1}, p_{t}^{2}, w_{t}\right)_{t=0}^{\infty}$.

- $\left(k_{t}^{j}, l_{t}^{j}\right)$ is a solution to profit maximization for firm $j, j=1,2$ for $\left(p_{t}^{1}, p_{t}^{2}, w_{t}\right)_{t=0}^{\infty}$.

In the present model, competitive equilibria are Pseudo-Pareto optimal allocations, i.e. solutions to the maximization of a "social" welfare function (see example 2.1, Kehoe, Levine and Romer (1992)) 


\subsection{The planner's optimum}

The Pseudo-Pareto optima are the set of solutions to the planner's problem as $\left(\mu_{a}, \mu_{b}\right)$ spans $[0,1] \times[0,1]$ :

$$
\begin{aligned}
\text { Max } & \mu_{a} N_{a} \sum_{t=0}^{\infty} \delta^{t} u_{a}\left(x_{a t}\right)+\mu_{b} N_{b} \sum_{t=0}^{\infty} \delta^{t} u_{b}\left(x_{b t}\right)+\left(1-\mu_{a}-\mu_{b}\right) N_{c} \sum_{t=0}^{\infty} \delta^{t} u_{c}\left(x_{c t}\right) \\
\text { s.t. } & N_{a} x_{a t}+N_{b} x_{b t}+N_{c} x_{c t}=F^{1}\left(k_{t-1}^{1}, l_{t}^{1}\right) \text { for all } t \\
& k_{t}^{1}+k_{t}^{2}=F^{2}\left(k_{t-1}^{2}, l_{t}^{2}\right) \text { for all } t \\
& l_{t}^{1}+l_{t}^{2}=z_{t}^{\eta} \text { for all } t \\
& k_{-1} \text { given }
\end{aligned}
$$

together with the side condition $z_{t}=k_{t-1}$.

For the case with no externalities, i.e. $\eta=0$, the solutions to the above program are interior as soon as $\omega_{i} \neq 0$ or $\theta_{i} \neq 0$ for $i=a, b, c$. As shown in Ghiglino and Olszak-Duquenne (2001) this is a consequence of the Inada conditions on preferences and technology.

Let $u$ be a social utility function defined by

$$
\begin{aligned}
u(x)= & \operatorname{Max} \mu_{a} N_{a} u_{a}\left(x_{a}\right)+\mu_{b} N_{b} u_{b}\left(x_{b}\right)+\left(1-\mu_{a}-\mu_{b}\right) N_{c} u_{c}\left(x_{c}\right) \\
& \text { s.t } N_{a} x_{a}+N_{b} x_{b}+N_{c} x_{c}=x
\end{aligned}
$$

Let $T(k, y, z)$ be the usual transformation function defined as the solution to

$$
\begin{array}{cl}
\text { Max } & F^{1}\left(k^{1}, l^{1}\right) \\
\text { s.t. } & F^{2}\left(k^{2}, l^{2}\right) \geq y \\
& k^{1}+k^{2}=k \\
& l^{1}+l^{2}=z^{\eta}
\end{array}
$$

Then the planner's problem is seen to be equivalent to

$$
\begin{aligned}
\operatorname{Max} & \sum_{t=0}^{\infty} \delta^{t} u\left(T\left(k_{t-1}, k_{t}, z_{t}\right)\right) \\
\text { s.t. } & F^{2}\left(k_{t-1}, z_{t}^{\eta}\right) \geq k_{t} \\
& k_{-1} \text { given }
\end{aligned}
$$

The solution depends on $z_{t}$ and $k_{-1}$. However, there is still the side condition $z_{t}=$ $k_{t-1}$. With the specification of production adopted through the paper the transformation function can be written as 


$$
T(k, y, z)=\left(z^{\eta}-y\right)^{\alpha}(k-\gamma y)^{1-\alpha}
$$

In the sequel we use the return function $V: R_{+} \times R_{+} \times R_{+} \rightarrow R$ defined by

$$
V(k, y, z)=u(T(k, y, z))
$$

The function $V$ is concave in $(k, y)$, because $u$ and $T$ are concave.

\subsection{The Euler conditions}

Using the return function $V(k, y, z)$ the maximization program can be written as

$$
\begin{aligned}
\text { Max } & \sum_{t=0}^{\infty} \delta^{t} V\left(k_{t}, k_{t+1}, z_{t+1}\right) \\
\text { s.t. } \quad\left(k_{t}, k_{t+1}\right) \in & D_{t} \\
& k_{-1} \text { given } \\
\text { Side condition: } & z_{t}=k_{t-1} .
\end{aligned}
$$

where $D_{t}$ is the set $\left\{\left(k_{t}, k_{t+1}\right) \mid F^{2}\left(k_{t}, z_{t}^{\eta}\right) \geq k_{t+1}\right\}$. Let $V_{1}(k, y, z)=\frac{\partial V(k, y, z)}{\partial k}$ and $V_{2}(k, y, z)=\frac{\partial V(k, y, z)}{\partial y}$.

In the present framework it is a standard result that the set of interior Pareto optima are the set of $\left\{k_{t}\right\}_{t}$ that satisfies the transversality condition $\lim _{t \rightarrow \infty} \delta^{t} V_{1}\left(k_{t}, k_{t+1}, k_{t}\right) k_{t}=0$ and are solutions to the system

$$
V_{2}\left(k_{t}, k_{t+1}, k_{t}\right)+\delta V_{1}\left(k_{t+1}, k_{t+2}, k_{t+1}\right)=0 \quad \forall t
$$

\subsection{Steady state in the capital good}

At a steady state, $k_{t}=k^{*}$ for every $t \geq 0$. The capital $k^{*}$ is implicitly defined by the equation

$$
V_{2}\left(k^{*}, k^{*}, k^{*}\right)+\delta V_{1}\left(k^{*}, k^{*}, k^{*}\right)=0
$$

In models with a unique consumption sector, aggregate steady state variables depend only on the technology. Indeed, using the definition of the return function, the Euler condition can be written as

$$
T_{2}\left(k^{*}, k^{*}, k^{*}\right)+\delta T_{1}\left(k^{*}, k^{*}, k^{*}\right)=0
$$

where $T_{1}(x, y, z)=\frac{\partial T(x, y, z)}{\partial x}$ and $T_{2}(x, y, z)=\frac{\partial T(x, y, z)}{\partial y}$. 
With the specification of production used in the present paper

$$
\begin{gathered}
T_{1}\left(k^{*}, k^{*}, k^{*}\right)=(1-\alpha)\left(\frac{k^{*^{\eta-1}}-1}{1-\gamma}\right)^{\alpha} \\
T_{2}\left(k^{*}, k^{*}, k^{*}\right)=-\left(\frac{k^{*^{\eta-1}}-1}{1-\gamma}\right)^{(\alpha-1)}\left[\alpha+\gamma(1-\alpha)\left(\frac{k^{*^{\eta-1}}-1}{1-\gamma}\right)\right]
\end{gathered}
$$

Some easy calculations gives the steady state capital as a function of the discount factor and the technology parameters only

$$
k^{*}=\left[\frac{(1-\alpha)(\gamma-\delta)}{\gamma-\alpha-\delta(1-\alpha)}\right]^{\frac{1}{1-\eta}}
$$

In the present paper, total labor supply is normalized to one. It is easy to see that in general $k^{*}$ represents the steady state capital normalized by the total labor supply. On the other hand, the aggregate consumption $x^{*}$ can also be obtained

$$
x^{*}=T\left(k^{*}, k^{*}, k^{*}\right)=k^{*}\left(k^{* \eta-1}-1\right)^{\alpha}(1-\gamma)^{1-\alpha}
$$

\subsection{The steady state in individual consumptions.}

At the steady state, aggregate capital depends on the total labor supply only. Indeed, as it does not depend on the return function the welfare weights are irrelevant, and both preferences and the way endowments are distributed among individuals do not matter. In more general models this is not true. In particular, when there are two consumption goods the steady state values of aggregate consumption depend on the individual welfare weights and therefore on the heterogeneity in preferences and endowments.

As opposed to aggregate variables, the steady state values of individual consumption do depend on individual characteristics through the welfare weights (note that at a steady state these are easy to compute). The exact relationship is provided by the following Lemma.

Lemma 1 At a steady state $k^{*}$ the individual demands are

$$
\left\{x_{i}{ }^{*}=\frac{x^{*}}{1-\gamma}\left[(\delta(1-\alpha)+\alpha-\gamma) \omega_{i}+(1-\delta)(1-\alpha) \theta_{i}\right]\right.
$$

where $x^{*}=k^{*}\left(k^{*^{\eta-1}}-1\right)^{\alpha}(1-\gamma)^{1-\alpha}$. 
Proof: The first order conditions associated to the individual program are

$$
\left\{\begin{array}{l}
\delta^{t} u_{i}^{\prime}\left(x_{i t}\right)=\nu_{i} p_{t}^{1} \quad \forall t, i=a, b \\
\sum_{t=0}^{\infty} p_{t}^{1} x_{i t}=\sum_{t=0}^{\infty} w_{t} \omega_{i}+\theta_{i} k_{-1}
\end{array}\right.
$$

where $\nu_{i}$ is the Lagrange multiplier associated to the constrained maximization problem. At a steady state $x_{i t}=x_{i}^{*}$ and $p_{t}^{1}=\delta^{t} p_{0}^{1}$ where $p_{0}^{1}$ is the price of the consumption good in period 0 . The budget constraint, together with the conditions on profit maximization, imply

$$
x_{i}^{*}=\frac{1-\delta}{p_{0}^{1}}\left(\sum_{t=0}^{\infty} w_{t} \omega_{i}+\theta_{i} k_{-1}\right)=\omega_{i} \frac{\partial F^{1}}{\partial l}\left(k_{1}^{*}, l_{1}^{*}\right)+(1-\delta) \theta_{i} k_{-1} \frac{\partial F^{1}}{\partial k}\left(k_{1}^{*}, l_{1}^{*}\right)
$$

The values of $k_{1}^{*}$ and $l_{1}^{*}$ are obtained by solving the system

$$
\left\{\begin{array}{l}
l_{1}^{*}+l_{2}^{*}=k^{*^{\eta}} \\
k_{1}^{*}+k_{2}^{*}=k^{*}=F^{2}\left(k_{2}^{*}, l_{2}^{*}\right)
\end{array}\right.
$$

and using the fact that at the optimum $l_{2}^{*}=\frac{k_{2}^{*}}{\gamma}$.

Q.E.D.

Lemma 1 implies that for given technology parameters and discount factor, steady state values in individual consumptions depend linearly on initial holdings in capital and labor endowments. This also means that there is a linear manifold of $\left(\theta_{i}, \omega_{i}\right)_{i=a, b, c}$ associated to each equilibrium allocation.

\section{Aggregate dynamics}

In the present general equilibrium model the reduced social utility function depends on the welfare weights. Furthermore, these weights depend on the equilibrium allocations which in turn depend on the initial conditions and on the distribution of individual endowments. When the welfare weights are continuous functions of the initial conditions, the dynamic and determinacy properties of the general equilibrium model with heterogeneous agents and those of the reduced model are identical. Ghiglino and Olszak-Duquenne (2001) have shown that when there are no externalities, continuity holds and the local dynamic and determinacy properties of the general equilibrium model are obtained from the properties of the planner's problem with the welfare weights fixed at the values associated to the steady state. Because of the externality the continuity property cannot be assumed here. However, it will be seen that the analysis of indeterminacy can be pursued to a large extent without this strong property. The relationship between the dynamic properties of the equilibrium path in the aggregate variables and the properties of the social utility function are first established. The use of these results in obtaining the properties of the disaggregated model is left for the next section. 


\subsection{The role of social risk aversion}

Near the steady state the behavior of the dynamic system is equivalent to the behavior of the linearized system. The dynamic properties of the steady state are then related to the eigenvalues of the matrix associated to the linearized system. In particular, the stability and the local determinacy properties of the steady state depends on how the modulus of the eigenvalues $\lambda_{1}$ and $\lambda_{2}$ compare to one.

Let the function $f$ be defined by

$$
f\left(k_{t}, k_{t+1}, k_{t+2}\right)=V_{2}\left(k_{t}, k_{t+1}, k_{t}\right)+\delta V_{1}\left(k_{t+1}, k_{t+2}, k_{t+1}\right) .
$$

The Euler equation

$$
V_{2}\left(k_{t}, k_{t+1}, k_{t}\right)+\delta V_{1}\left(k_{t+1}, k_{t+2}, k_{t+1}\right)=0
$$

can be linearized at the steady state $\left(k^{*}, k^{*}, k^{*}\right)$ to give

$$
\frac{\partial f}{\partial k_{t+2}}\left(k^{*}, k^{*}, k^{*}\right)\left(k_{t+2}-k^{*}\right)+\frac{\partial f}{\partial k_{t+1}}\left(k^{*}, k^{*}, k^{*}\right)\left(k_{t+1}-k^{*}\right)+\frac{\partial f}{\partial k_{t}}\left(k^{*}, k^{*}, k^{*}\right)\left(k_{t}-k^{*}\right)=0
$$

This equation describes the dynamic behavior of $\left\{k_{t}\right\}_{t}$ near $k^{*}$. The resolution of the characteristic equation

$$
\frac{\partial f}{\partial k_{t+2}}\left(k^{*}, k^{*}, k^{*}\right) p^{2}+\frac{\partial f}{\partial k_{t+1}}\left(k^{*}, k^{*}, k^{*}\right) p+\frac{\partial f}{\partial k_{t}}\left(k^{*}, k^{*}, k^{*}\right)=0
$$

for $p$ gives the eigenvalues associated to the dynamical system. As there is only one consumption good, for a given technology and aggregate labor the steady state value of aggregate capital is uniquely determined and does not depend on the characteristics of the consumers. However, the stability properties of the steady state do depend on the social utility function and therefore on the individual characteristics. The following notion need to be introduced.

Definition 2 Let $u$ be the social utility function, $u: R_{+} \rightarrow R$, and $R(x)$ the risk aversion, $R(x)=-\frac{u^{\prime \prime}(x)}{u^{\prime}(x)}$. Let $\rho$ be the inverse of the risk aversion, $\rho=-\frac{1}{R}=\frac{u^{\prime}(x)}{u^{\prime \prime}(x)}$.

Note that $\rho$ is negative and that $\rho$ close to zero indicates a very high degree of curvature of the utility function.

For a given externality, discount factor and technology parameters, the eigenvalues depend on the inverse of the risk aversion of the social utility function, $\rho$, through the equality $V(k, y, z)=u(T(k, y, z))$. Indeed, we have the following result.

Lemma 2 The eigenvalues associated to the dynamic system expressed in terms of the inverse of risk aversion $\rho$ are

$$
p_{1,2}=\frac{1}{2}\left[-B \pm \sqrt{B^{2}-4 C}\right]
$$


with

$$
\begin{gathered}
B=\frac{\rho\left(T_{22}+\delta\left(T_{11}+T_{13}\right)\right)+\delta T_{1}\left((1+\delta) T_{1}+T_{3}\right)}{\delta\left(-\delta T_{1}^{2}+\rho T_{12}\right)} \\
C=\frac{1}{\delta}\left[1+\frac{-\delta T_{1} T_{3}+\rho T_{23}}{-\delta T_{1}^{2}+\rho T_{12}}\right]
\end{gathered}
$$

where $T_{i j}=T_{i j}\left(k^{*}, k^{*}, k^{*}\right), i, j=1,2,3$ are the second order derivatives of $T$.

Proof: The eigenvalues, which are the solutions of the characteristic equation $(C E)$, are given by

$$
p_{1,2}=\frac{1}{2}\left[-B \pm \sqrt{B^{2}-4 C}\right]
$$

with $B=\left(\left.\frac{\partial f}{\partial k_{t+1}}\right|_{k^{*}}\right)\left(\left.\frac{\partial f}{\partial k_{t+2}}\right|_{k^{*}}\right)^{-1}$ and $C=\left(\left.\frac{\partial f}{\partial k_{t}}\right|_{k^{*}}\right)\left(\left.\frac{\partial f}{\partial k_{t+2}}\right|_{k^{*}}\right)^{-1}$

Let $V_{i j}=\frac{\partial^{2} V}{\partial k_{i} \partial k_{j}}\left(k^{*}, k^{*}, k^{*}\right)$. The definition of $f$ implies that

$$
\left.\frac{\partial f}{\partial k_{t+2}}\right|_{k^{*}}=\delta V_{12},\left.\frac{\partial f}{\partial k_{t+1}}\right|_{k^{*}}=\delta\left(V_{11}+V_{13}\right)+V_{22} \text { and }\left.\frac{\partial f}{\partial k_{t}}\right|_{k^{*}}=V_{12}+V_{23}
$$

and then $B=\frac{\delta\left(V_{11}+V_{13}\right)+V_{22}}{\delta V_{12}}$ and $C=\frac{V_{12}+V_{13}}{\delta V_{12}}$

At the steady state and for $i, j=1,2,3, V(k, y, z)=u(T(k, y, z))$ implies that

$$
\begin{array}{r}
V_{i j}\left(k^{*}, k^{*}, k^{*}\right)=u^{\prime \prime}\left(T\left(k^{*}, k^{*}, k^{*}\right)\right) T_{i} T_{j}+u^{\prime}\left(T\left(k^{*}, k^{*}, k^{*}\right)\right) T_{i j} \\
\Longrightarrow \frac{\left.V_{i j}\left(k^{*}, k^{*}, k^{*}\right)\right)}{u^{\prime \prime}\left(T\left(k^{*}, k^{*}, k^{*}\right)\right)}=T_{i} T_{j}+\frac{u^{\prime}\left(T\left(k^{*}, k^{*}, k^{*}\right)\right)}{u^{\prime \prime}\left(T\left(k^{*}, k^{*}, k^{*}\right)\right)} T_{i j}=T_{i} T_{j}+\rho T_{i j}
\end{array}
$$

Expressions (1) and (2) and the Euler equation $T_{2}=-\delta T_{1}$ lead to the announced values for $B$ and $C$.

Q.E.D.

The result of Lemma 2 is represented in Fig. 1 where $p_{1}$ is the eigenvalue with the smallest modulus, i.e. $\left|p_{1}\right|<\left|p_{2}\right|$.

\section{INSERT Fig. 1 HERE}

A property which plays an important role in the subsequent developments is that at most one of the two graphs $p_{1}(\rho)$ and $p_{2}(\rho)$ intersect the horizontal line drawn at -1 . This is a consequence of the fact that the branches of $p_{i}(\rho)$ are monotonous as shown in Fig. 1. As the stability and determinacy is linked to the modulus of the eigenvalues (with 1 as the critical value), the previous fact implies that only three cases are feasible: 
Case $A$ Neither $p_{1}$ nor $p_{2}$ intersect the line $p=-1$. The steady state is always determinate and stable whatever the value of $\rho$.

Case $B$ The eigenvalue $p_{1}$ intersects the line $p=-1$ while $p_{2}<-1$. Stability and instability are possible but indeterminacy is ruled out.

Case $C$ The eigenvalue $p_{2}$ intersects the line $p=-1$ while $1>p_{1}>-1$. Both (determinate) stability and indeterminacy are possible.

These remarks lead to the following statement:

Corollary 1 For any given set of admissible parameters $(\alpha, \gamma, \eta, \delta)$, there exists a value of the risk aversion of the social utility function, $R_{0}$, such that for all economies with a higher curvature, $R>R_{0}$, the steady state of the reduced model is stable and determinate.

The graph $p_{i}(\rho)$ is a representation of one member of a family of functions. The two families, $i=1$ and $i=2$, are obtained when the quadruples $(\alpha, \gamma, \eta, \delta)$ span the set of feasible values. A change in one of the parameters is likely to modify the graph so that different cases for (determinate) stability and indeterminacy of the steady state arise depending on the quadruple $(\alpha, \gamma, \eta, \delta)$. A complete analysis would require to know how these graphs are shifted and modified when the parameters change. The dependance is complex and the effects of each one of the parameters are hardly separable.

The following lemma gives sufficient conditions on the parameters for the existence of a (negative) solution $\rho_{c}$ to the equation $p_{i}=-1$ for $i=1$ or $i=2$. In other words it gives open sets of technology parameters and time discount factor such that changes in the curvature may bring a change in the dynamic behavior of the economy (case B) or/and in determinacy (case $\mathrm{C}$ ).

Lemma 3 (i) If

$$
\begin{gathered}
\frac{1}{3}<\alpha<\frac{1}{2} ; \gamma>1-2 \alpha \quad ; \quad \eta>\bar{\eta}=\frac{2 \alpha+\gamma-1}{1-\gamma} \\
\text { or } \\
\alpha>\frac{1}{2} ; \gamma<1-\alpha \quad ; \quad \eta>\bar{\eta}
\end{gathered}
$$

then there exists $\bar{\delta}$ such that for all $\delta$ in $\left.I_{\delta}=\right] \bar{\delta}, 1\left[\right.$, there exists $\rho_{c}<0$ such that $p_{2}\left(\rho_{c}\right)=$ -1 . There is indeterminacy for $\rho<\rho_{c}$ and determinate stability otherwise (case $C$ ).

(ii) If

$$
\left.\alpha<\frac{1}{3} \quad \text { and } \quad \gamma \in\right] \frac{\alpha(1-2 \alpha)}{\sqrt{(1-\alpha)^{2}+(1-2 \alpha)^{2}}}, \alpha[
$$




$$
\left.\frac{1}{3}<\alpha<\frac{1}{2} \quad \text { and } \quad \gamma \in\right] \frac{\alpha(1-2 \alpha)}{\sqrt{(1-\alpha)^{2}+(1-2 \alpha)^{2}}}, 1-2 \alpha[
$$

then there exist $\delta_{c}$ and $\delta_{c c}$ in ] 0,1 [ such that for all $\delta$ in $\left.I_{\delta c}=\right] \delta_{c}, \delta_{c c}\left[\right.$, there exists $\rho_{c}<0$ such that $p_{1}\left(\rho_{c}\right)=-1$ or $p_{2}\left(\rho_{c}\right)=-1$ (cases $B$ or $\left.C\right)$.

Proof : See the appendix.

Remark: The conditions given for $(i)$ are necessary and sufficient while those for $(i i)$ are only sufficient (see the proof for details).

Lemma 3 is illustrated by some numerical examples.

Case $A$ No negative solution. Let $\alpha=0.3$ and $\gamma=0.12<\bar{\gamma}=0.1488$. Then $\rho_{c}=2.2145>$ 0 .

Case $B A$ solution to $p_{1}=-1$. Let $\alpha=0.15, \gamma=0.13$ and $\eta=0.1$. Then $I_{\delta c}=$ ]0.1857, 0.2973[. For $\delta=0.2, \rho_{c}=-18.84$. When $\rho \longrightarrow-\infty, p_{1} \longrightarrow-1.25$ and $p_{2} \longrightarrow-3.48$.

Case $C A$ solution to $p_{2}=-1$. Let $\alpha=0.7, \gamma=0.2$ and $\eta=0.8$. Then $\left.I_{\delta}=\right] 9259,1[$. For $\delta=0.95, \rho_{c}=-0.3736$. Then, when $\rho \longrightarrow-\infty, p_{1} \longrightarrow-0.1278$ and $p_{2} \longrightarrow$ -0.8471 .

Lemma 3 will be applied to the dynamics of the disaggregate economy in Section 5 and Section 6.

\subsection{Turnpike properties}

Lemma 3 concerns the dynamic properties of the steady state when the technology, the welfare weights (or the individual preferences and endowments) and the time discount factor are fixed parameters. A classical result concerns the asymptotic stability properties when the time discount tends to zero, i.e. $\delta \longrightarrow 1$.

\section{Lemma 4}

i) If $\alpha<\frac{1}{2}$ and $\gamma<1-2 \alpha$ then for given technology, preferences and initial endowments there exists $\delta_{s}<1$ such that the steady state is stable for $\delta_{s}<\delta<1$.

ii) If $\alpha$ and $\gamma$ satisfy the hypothesis $(i)$ of Lemma 3, then there exists a value $\eta=\bar{\eta}$ such that:

- if $\eta<\bar{\eta} \Longrightarrow$ there exists $\delta_{s}$ such that the steady state is stable for all $\delta>\delta_{s}$. 
- if $\eta>\bar{\eta} \Longrightarrow$ the stability and determinacy can be influenced by the curvature of the social utility function for all $\delta>\bar{\delta}$, with $\bar{\delta}$ defined in Lemma 3.

iii) If $\alpha>\frac{1}{2}$ and $\gamma>1-\alpha$, there exists $\delta_{s}$ such that the steady state is stable for all $\delta>\delta_{s}$.

Proof: A straightforward application of Lemma 3.

Q.E.D.

Remark: For a weak externality the steady state always becomes stable as $\delta \longrightarrow 1$. This result does not hold for a sufficiently strong externality.

In general equilibrium convex models the limit point depends on the distribution of initial capital and labor, even when the turnpike property holds. However, Yano (1984, 1991, 1998) shows that the sensitivity to the initial capital shares tends to disappear as the time discount factor $\delta$ approaches one. A similar result concerning the steady states holds in the present model. Note that the limit points concerning the individual consumptions depend on the individual endowment in labor.

Lemma 5 When $\delta$ is sufficiently close to one the steady states associated to different distributions of individual holdings of initial capital lie in a neighborhood of $\overline{k^{*}}=(1-$ $\alpha)^{\frac{1}{1-\eta}}$ and this neighborhood shrinks as $\delta \rightarrow 1$. Similarly, the individual steady state consumptions lie close to

$$
\overline{x_{i}^{*}}=\alpha^{\alpha}[(1-\alpha)(1-\gamma)]^{1-\alpha} \omega_{i}
$$

Proof: From Lemma 1 with straightforward calculations.

When agents differ only in respect to their shares in the initial capital, as the discount factor tend to one their individual consumptions converge to a common value. On the other hand, for a given discount their relative distance is given by

$$
\frac{x_{i}^{*}-x_{j}^{*}}{x^{*}}=\frac{(1-\alpha)(1-\delta)}{(1-\gamma)}\left(\theta_{i}-\theta_{j}\right)
$$

\section{Indeterminacy in the heterogenous agents econ- omy}

The aim of this section is to prove local indeterminacy in the heterogeneous agents economy under scrutiny. The analysis of the previous section relates the dynamic behavior of 
the aggregate variables to the curvature of the social utility function, the time discount factor and the technology parameters. Heterogeneity across consumers affects the welfare weights and then the social utility function. Therefore, determinacy and stability of the aggregate steady state are expected to depend on the heterogeneity. Finally, indeterminacy of the solutions to the planner's problem implies that there exist disaggregate economies with indeterminate competitive equilibria.

Proposition 1 There exists open sets of parameters $\alpha, \gamma, \eta, \delta$ and open sets of preferences such that local indeterminacy around the competitive steady state occurs for some distributions of initial endowments or capital shares but not for others. The result holds also if the agents have identical preferences and labor endowments.

Proof: $i$ ) In the appendix it is shown that the value of the curvature at the steady state is given by

$$
\rho\left(\theta_{a}, \theta_{b}, \theta_{c}, \omega_{a}, \omega_{b}, \omega_{c}\right)=N_{a} \frac{u_{a}^{\prime}}{u_{a}^{\prime \prime}}\left(x_{a}^{*}\left(\theta_{a}, \omega_{a}\right)\right)+N_{b} \frac{u_{b}^{\prime}}{u_{b}^{\prime \prime}}\left(x_{b}^{*}\left(\theta_{b}, \omega_{b}\right)\right)+N_{c} \frac{u_{c}^{\prime}}{u_{c}^{\prime \prime}}\left(x_{c}^{*}\left(\theta_{c}, \omega_{c}\right)\right)
$$

Without loss of generality, we may assume that preferences are identical so that the subscripts can be dropped and that $N_{a}=N_{b}=N_{c}=1$.

ii) Assume that the coefficients $\alpha, \gamma$ and $\eta$ satisfy the assumption in case $(i)$ of Lemma 3 , and let $\delta$ be in $I_{\delta}$. According to Lemma 3, these assumptions imply that $\rho_{c}<0$ and that indeterminacy for the reduced model occurs for any $\rho_{I}<\rho_{c}$.

Since there are no structural constraints on the first and second derivatives of the individual utility function, except the usual sign conditions, the previous expression implies that $\rho_{I}$ can be obtained with a suitable choice of preferences. Furthermore, since $\rho(\theta, \omega)$ depends on the derivatives of the individual utility functions evaluated at different points, the result still holds when the preferences for all agents are identical.

Indeterminacy in the aggregate dynamics imply indeterminacy in the disaggregated model with heterogenous agents. Indeed, given the aggregate initial stock $k_{-1}$ and the welfare weights $\left(\mu_{a}, \mu_{b}\right)$ there is a continuum of paths $\left(k_{t}\right)_{t=0}^{\infty}$ converging to the steady state. For each of these paths, the first order condition associated to

$$
\begin{aligned}
& \operatorname{Max} \mu_{a} u_{a}\left(x_{a t}\right)+\mu_{b} u_{b}\left(x_{b t}\right)+\left(1-\mu_{a}-\mu_{b}\right) u_{c}\left(x_{c t}\right) \\
& \text { s.t } x_{a t}+x_{b t}+x_{c t}=T\left(k_{t-1}, k_{t}, k_{t-1}\right)
\end{aligned}
$$

gives the individual consumption allocations $\left(x_{a t}\right)_{t=0}^{\infty},\left(x_{b t}\right)_{t=0}^{\infty}$ and $\left(x_{c t}\right)_{t=0}^{\infty}$. Finally, $\left(\theta_{a}, \theta_{b}\right)$ is obtained from the individual budget constraint and the values of the prices and the wages. Due to the indeterminacy there is an open interval of feasible $\left(\theta_{a}, \theta_{b}\right)$ with the same $k_{-1}$ and $\left(\mu_{a}, \mu_{b}\right)$. On the other hand, in a neighborhood of the steady state, a small perturbation of the welfare weights will only slightly affect the path $\left(k_{t}\right)_{t=0}^{\infty}$. Due to the continuity of the functions involved and the fact that prices converge to the steady state price, the interval of feasible $\left(\theta_{a}, \theta_{b}\right)$ is also only slightly affected. Therefore, there is an open set of $\left(\theta_{a}, \theta_{b}\right)$ such that for each element in this set there is a continuum of equilibrium paths. Therefore the steady state is indeterminate in the individual variables. 
iii) As in ii) assume that the parameters fulfill the conditions stated in i) of Lemma 3. Consider now a redistribution of initial capital shares such that all agents becomes identical. The economy trivially admits a representative agent. In this situation, for any $\rho_{s}>\rho_{c}$ the steady state is stable (and determinate). Since, there are no structural constraints on the first and second order derivatives of the individual utility function, except the usual sign conditions, the preferences chosen in $i$ ) can be perturbed as to satisfy also $\rho=\rho_{s}$.

Q.E.D.

In Proposition 1, preferences are not bound to belong to some specific class. In particular, if preferences are assumed to be CES then the derivatives of all orders are given functions and the proof fails. However, it can still be shown that the result holds in this case, the argument being similar to Ghiglino and Olszak-Duquenne (2001) (for the details see Ghiglino and Olszak-Duquenne (2000)).

It should be pointed out that Proposition 1 does not concern the effects of all redistributions of initial endowments on dynamics and determinacy. Indeed, this would require to be able to decide whether the steady state of the general disaggregated model is determinate or indeterminate, a much harder task. When the welfare weights are continuous functions of the initial conditions, the local dynamic properties of the general equilibrium model are indeed equivalent to the dynamic properties of the aggregate "optimal growth" model with the welfare weights fixed at the steady state values. There might be conditions ensuring that such strong continuity properties also hold in the present model but we do not have a formal proof.

\section{When heterogeneity works against indeterminacy}

Heterogeneity is one of the main macroeconomic indicators of the microeconomic structure of the economy. Our aim in this section is to link indeterminacy to heterogeneity. There are several difficulties. The first is that heterogeneity is a concept that is not uniquely defined. When agents have identical preferences, a good indicator of heterogeneity is the spread of initial endowments in capital shares and labor. If we furthermore assume that only shares in initial capital (or labor endowments) differ, the agents can be distributed on the real line. An homogeneous economy is then an economy that has a distribution of shares (or labor endowments) picked around some value, while in an heterogenous economy these are widely spread. This notion agrees with the definition of heterogeneity given in Herrendorf et al. (2000), Definition 3. In the present model, there are three types of agents, so that an heterogeneous economy has an equal number of agents of each type, while an homogeneous economy has mostly all agents of the same type.

That heterogeneity so defined may have an effect on indeterminacy is a consequence of Proposition 1. In fact this link is expected to hold under general conditions (see Corollary 1). However, it may also be seen that the usual axioms on preferences don't limit the sign 
of the effect. The reason is that the occurrence of indeterminacy depends on the third and other high order derivatives of the utility functions. Standard assumptions on preferences do not put any limitation on these and empirical data is also lacking. However, these higher order derivatives are linked to risk aversion. And the properties of risk aversion are better known from an empirical point of view.

Several results can be obtained. When relative risk aversion is equal across individuals and constant, i.e., when preferences are identical across individuals and CES, heterogeneity is shown to play no role on indeterminacy or instability. When risk aversion is a strictly concave function, homogeneity is a source of indeterminacy or instability. When risk aversion is a strictly convex function homogeneity is still a source of indeterminacy or instability, provided the second derivative of risk aversion is sufficiently small. As data is compatible with concave or slightly convex risk aversion, one may think that in realistic models heterogeneity may help to restore determinacy in economies with external effects. This agrees and extend the results in Herrendorf et al. (2000).

Proposition 2 Assume that agents are characterized by identical preferences with risk aversion $R(x)$. If $R(x)$ is a strictly concave function, then homogeneity is a source of indeterminacy or instability. The same result hold if risk aversion is a strictly convex function with $2 R^{\prime 2}(x) / R(x)>R^{\prime \prime}(x)$, while if the latter inequality is reversed, heterogeneity becomes a source of indeterminacy or instability. Finally, if the parameters $\alpha, \gamma, \eta, \delta$ satisfy the conditions in Lemma 3 (i), then all the results above concern indeterminacy (and not instability).

Proof: Assume that preferences and labor endowments are identical across consumers, $u_{a}(x)=u_{b}(x)=u_{c}(x)=v(x)$ and $\omega_{a}=\omega_{b}=\omega_{c}=\omega$. The proof of Proposition 1 shows that the reaction of the curvature of the social utility function to a change in individual consumption depends on the inverses of the individual risk aversion. Corollary 1 , and the discussion therein, show that in order to analyze the effect of heterogeneity on indeterminacy or instability it is sufficient to compare two stylized situations: 1) The economy is homogenous, there is one agent of each type, $N_{a}=N_{b}=N_{c}=1$; and 2) there is only one type of consumer, i.e. $N_{b}=3$. The exact value of the individual capital shares is irrelevant for the proof, as long as $x_{a}^{*}\left(\theta_{a}, \omega\right)<x_{b}^{*}\left(\theta_{b}, \omega\right)<x_{c}^{*}\left(\theta_{c}, \omega\right)$. We take $\theta_{a}=1 / 6, \theta_{b}=2 / 6$ and $\theta_{c}=3 / 6$. Consequently, we need to compare

$$
\rho_{h e}=\frac{v^{\prime}}{v^{\prime \prime}}\left(x^{*}(1 / 6, \omega)\right)+\frac{v^{\prime}}{v^{\prime \prime}}\left(x^{*}(2 / 6, \omega)\right)+\frac{v^{\prime}}{v^{\prime \prime}}\left(x^{*}(3 / 6, \omega)\right)
$$

with

$$
\rho_{h o}=3 \frac{v^{\prime}}{v^{\prime \prime}}\left(x^{*}(1 / 3, \omega)\right)
$$

Three cases need to be analyzed depending on the properties of risk aversion. 
(i) When preferences are identical across consumers and risk aversion is constant, $R_{j}(x)=$ $R$ so that $v^{\prime}\left(x_{j}\right) / v^{\prime \prime}\left(x_{j}\right)=-1 / R$, the effect on the parameter $\rho$ is additive and weighted by the number of consumers. In this case, there is no impact on either stability or determinacy of the steady state and heterogeneity is neutral.

(ii) Assume that preferences are such that risk aversion is a concave function over the relevant range. Since $\left(v^{\prime}(x) / v^{\prime \prime}(x)\right)^{\prime}=(-1 / R(x))^{\prime}=-\left(R^{2}(x)\right)^{-1} R^{\prime}(x)$ we obtain

$$
\left(v^{\prime}(x) / v^{\prime \prime}(x)\right)^{\prime \prime}=\left(-\left(R^{2}(x)\right)^{-1} R^{\prime}(x)\right)^{\prime}=2\left(R^{3}(x)\right)^{-1}\left(R^{\prime}(x)\right)^{2}+\left(-\left(R^{2}(x)\right)^{-1} R^{\prime \prime}(x)\right)>0
$$

i.e., a strictly convex function. In this case,

$$
\rho_{h o}<\rho_{h e}<0
$$

and indeterminacy is more likely in the homogenous economy then in the heterogenous economy.

(iii) Assume instead that risk aversion is a convex function over the relevant range. In this case, provided $\left|2\left(R^{3}(x)\right)^{-1}\left(R^{\prime}(x)\right)^{2}\right|<\left|-\left(R^{2}(x)\right)^{-1} R^{\prime \prime}(x)\right|$ or $2(R(x))^{-1}\left(R^{\prime}(x)\right)^{2}<R^{\prime \prime}(x)$, we obtain

$$
\left(v^{\prime}(x) / v^{\prime \prime}(x)\right)^{\prime \prime}=2\left(R^{3}(x)\right)^{-1}\left(R^{\prime}(x)\right)^{2}+\left(-\left(R^{2}(x)\right)^{-1} R^{\prime \prime}(x)\right)<0
$$

i.e., a strictly concave function. As a conclusion,

$$
\rho_{h e}<\rho_{h o}<0
$$

and indeterminacy is more likely in the heterogenous economy. On the other hand, if $2(R(x))^{-1}\left(R^{\prime}(x)\right)^{2}>\left|R^{\prime \prime}(x)\right|$ then indeterminacy is more likely in the homogenous economy.

Finally, if the parameters satisfy the conditions in Lemma 3 (i) then the steady state is indeterminate for any sufficiently small social curvature. In this case, the results obtained so far are related to indeterminacy.

Q.E.D.

The above results are consistent with the view that the importance of indeterminacy in models with externalities are overstated in the literature. Indeed, we have found plausible circumstances for which heterogeneity is a factor that tends to neutralize the externality as a source of indeterminacy. It should be pointed out that in our model, heterogeneity in individual productivity are not explicitly taken into account. However, as we allow for heterogeneity in labor endowments, our model can be reinterpreted as to include different levels of individual labor productivity. 


\section{References}

1. BEWLEY, T. (1982). "An integration of equilibrium theory and turnpike theory", Journal of Mathematical Economics, 10, 233-267.

2. BOLDRIN, M., and A. RUSTICHINI (1994). "Growth and indeterminacy in dynamic models with externalities", Econometrica, 62, 323-342.

3. GHIGLINO, C., (2002). "Introduction to Economic Growth and General Equilibrium", Journal of Economic Theory, 105 , 1-17.

4. GHIGLINO, C., and M. OLSZAK-DUQUENNE (2001). "Inequalities and fluctuations in a dynamic general equilibrium model", Economic Theory, 17, 1-24

5. GHIGLINO, C., and M. OLSZAK-DUQUENNE, (2000). "Growth with many agents and externalities", Department of Econometrics, University of Geneva.

6. GHIGLINO, C., and G. SORGER (2002). "Indeterminacy, wealth distribution and poverty traps", Journal of Economic Theory, 105, 120-139.

7. GRANDMONT, J.-M., (1992). "Transformation of the commodity space, behavioral heterogeneity and the aggregation problem", Journal of Economic Theory, 57, $1-35$.

8. HERRENDORF, B., R. WALDEMANN and A. VALENTINYI (2000). "Ruling out multiplicity and indeterminacy: the role of heterogeneity", Review of Economic Studies, 67, 295-307.

9. KEHOE, T. J., D. K. LEVINE, and P. M. ROMER (1990). "Determinacy of equilibrium in dynamic models with finitely many consumers", Journal of Economic Theory, 50, 1-21.

10. KEHOE, T. J., D. K. LEVINE, and P. M. ROMER (1992). "On characterizing equilibria of economies with externalities and taxes as solutions to optimization problems", Economic Theory, 2, 43-68.

11. MATSUYAMA, K. (1991) "Increasing returns, industrialization, and indeterminacy of equilibrium", Quarterly Journal of Economics, 104, 617-650.

12. SANTOS, M. (1992)."Differentiability and comparative analysis in discrete-time infinite-horizon optimization", Journal of Economic Theory, 57, 222-229.

13. YANO, M. (1984). "The turnpike of dynamic general equilibrium path and its insensitivity to initial conditions," Journal of Mathematical Economy, 13, 235-254.

14. YANO, M. (1991). "Temporary transfers in a simple dynamic general equilibrium model", Journal of Economic Theory, 54, 372-388. 
15. YANO, M. (1998). "On the dual stability of a von Neumann facet and the inefficacy of temporary fiscal policy", Econometrica, 66, 427-451.

\section{Appendix}

\subsection{Proof of Lemma 3: Existence of the roots}

First, remark that $p_{1}=-1$ and $p_{2}=-1$ are satisfied simultaneously only if $p_{1}=$ $\frac{-B+\sqrt{B^{2}-4 C}}{2}=p_{2}=\frac{-B-\sqrt{B^{2}-4 C}}{2}=-1$ (the choice of the sign depends on the convention $\left.\left|p_{1}\right|<\left|p_{2}\right|\right)$. This implies $\sqrt{B^{2}-4 C}=B-2=0$ and $C=1$. This is a non generic situation.

Now, $p_{1,2}=-1 \Leftrightarrow \pm \sqrt{B^{2}-4 C}=-(B-2)$. This implies $B-C=1$. Using Lemma 2 which gives the values of $B$ and $C$ in terms of $\rho$, we obtain

$$
B-C=1 \Leftrightarrow \frac{\left(T_{22}-T_{12}-T_{23}+\delta\left(T_{11}+T_{13}\right)\right) \rho+\delta T_{1}\left(\delta T_{1}+2 T_{1}+2 T_{3}\right)}{\delta\left(\rho T_{12}+T_{1} T_{2}\right)}=1
$$

Solving for $\rho$ gives the solution

$$
\rho_{c}=\frac{-2 \delta T_{1}\left(T_{1}(1+\delta)+T_{3}\right)}{T_{22}-T_{12}-T_{23}+\delta\left(T_{11}+T_{13}-T_{12}\right)}
$$

Some tedious calculations show that

$$
\rho_{c}=\alpha \delta x^{*} \frac{Q(\delta)}{f(\delta)}
$$

with

$$
\begin{gathered}
Q(\delta)=\delta(1-\alpha)(1+\eta)+(1-\alpha)+\eta(\alpha-\gamma) \\
f(\delta)=a \delta^{2}+b \delta+c
\end{gathered}
$$

and

$$
\left\{\begin{array}{l}
a=(1-\alpha)(1-2 \alpha)(1+\eta) \\
b=-[\alpha(1-\eta)(1-2 \alpha)+\gamma(1+\eta)(2-3 \alpha)] \\
c=\gamma[\gamma(1+\eta)+\alpha(1-\eta)]
\end{array}\right.
$$

The value $\rho_{c}$ is an acceptable solution for the equation $p_{i}=-1$ provided it is strictly negative (as it is the ratio of the first derivative $u^{\prime}$ and the second derivative $u^{\prime \prime}$ evaluated at the steady state).

As the numerator is always strictly positive, $f(\delta)$ need to be negative. Since, the concavity of $f$ plays a role in the proof, the two cases $\alpha<\frac{1}{2}$ and $\alpha>\frac{1}{2}$ should be treated separately. 
Case A: $\alpha<\frac{1}{2}$

In this case, $f$ is convex, with $a>0, b<0$ and $c>0$ implying that $f(0)>0$. There are 2 possibilities to obtain $f(\delta)<0$.

- Existence of an interval included in $] 0,1[$ on which $f$ is negative. The sufficient conditions are a positive discriminant for $f$ with roots between 0 and 1 . As the analysis is tedious, we give conditions on the parameters such that: $f(1)>0$, the critical point of $f$ is positive and the product of the roots lies between 0 and 1 . These properties are sufficient to prove the result.

- Existence of a value $\bar{\delta}$ in $] 0,1[$ such that $f(\delta)<0$ on $] \bar{\delta}, 1[$. This requires a positive discriminant and $f(1)<0$.

\section{a) Sign of the discriminant $\Delta$ of $f$}

$\Delta$ can be considered as a polynomial function of degree 2 in $\eta$ :

$$
\Delta(\eta)=a^{\prime} \eta^{2}+b^{\prime} \eta+c^{\prime}
$$

with:

$$
\left\{\begin{array}{l}
a^{\prime}=[\gamma(1-\alpha)-(\alpha-\gamma)(1-2 \alpha)]^{2}+4 \gamma(1-\alpha)(1-2 \alpha)(\alpha-\gamma) \\
b^{\prime}=2\left[\gamma^{2}\left((1-\alpha)^{2}+(1-2 \alpha)\right)^{2}-\alpha^{2}\left(1-2 \alpha^{2}\right)\right] \\
\left.c^{\prime}=(1-2 \alpha)^{2}(\alpha+\gamma)^{2}+(1-\alpha)^{2} \gamma^{2}\right)
\end{array}\right.
$$

If (and only if) all the coefficients are positive then the function $\Delta(\eta)$ is positive for all values of $\eta$. $a^{\prime}$ is clearly positive under the assumption $\alpha<\frac{1}{2}$. $b^{\prime}$ is positive only if $\gamma>\frac{\alpha(1-2 \alpha)}{\sqrt{(1-\alpha)^{2}+(1-2 \alpha)^{2}}}=\bar{\gamma}$. As $\bar{\gamma}<1-2 \alpha$ the ratio $\frac{\bar{\gamma}}{1-2 \alpha}$ is always smaller than 1. Finally, $c^{\prime}$ is always positive for all value of $\alpha$ and $\gamma$. Under these assumptions, the discriminant $\Delta$ being positive for all values of $\eta$, there exist two roots $\delta_{c}$ and $\delta_{c c}$.

b) Position of the roots $\delta_{c}$ and $\delta_{c c}$ in relation with the interval ] 0,1 [.

Since the critical point $-\frac{b}{2 a}$ is positive, $f$ is convex and $f(0)>0$.both roots are positive. In order to find their position relative to 1 , the sign of $f(1)$ as well as the value of the product of the roots $\delta_{c} \delta_{c c}$ need to be analyzed.

b.1) Sign of $f(1)$

By definition, $f(1)=(1-2 \alpha-\gamma)[\eta(1-\gamma)+1-2 \alpha-\gamma]$. Then $f(1)$ is positive if:

- $(1-2 \alpha-\gamma)>0$ and $\eta(1-\gamma)+1-2 \alpha-\gamma>0$, which is equivalent to $1-2 \alpha-\gamma>0$ giving $1-2 \alpha>\gamma$.

- $(1-2 \alpha-\gamma)<0$ and $\eta(1-\gamma)+1-2 \alpha-\gamma<0$, which is equivalent to $1-2 \alpha<\gamma$ and $\eta<-\frac{1-2 \alpha-\gamma}{1-\gamma}:=\bar{\eta}$. However, as $\gamma<\alpha$. the first condition implies $\alpha>\frac{1}{3}$.

The $f(1)$ is negative if: 
$\gamma>1-2 \alpha$ (implying $\alpha>\frac{1}{3}$ ) and $\eta>\bar{\eta}$.

The results regarding $f(1)$ can be reorganized as:

- If $0<\alpha<\frac{1}{3}$ then $\alpha<1-2 \alpha$, so that $\gamma<\alpha<1-2 \alpha$

$\Rightarrow f(1)>0$

- If $\frac{1}{3}<\alpha<\frac{1}{2}$, two possibilities arise:

(i) $\gamma<1-2 \alpha \quad \Rightarrow f(1)>0$

(ii) $1-2 \alpha<\gamma<\alpha$ : the sign of $f(1)$ depends on the externality: $f(1)$ is positive for $\eta<\bar{\eta}$ and negative for $\eta>\bar{\eta}$

\section{b.2) Study of the product $\delta_{c} \delta_{c c}$}

The product of the roots $\delta_{c} \delta_{c c}$ is equal to $\frac{c}{a}$. Then the following relation holds:

$$
\frac{c}{a}<1 \Longleftrightarrow \eta>\frac{\gamma(\alpha+\gamma)-(1-\alpha)(1-2 \alpha)}{\gamma(\alpha-\gamma)+(1-\alpha)(1-2 \alpha)}=\tilde{\eta}
$$

When $\gamma<1-2 \alpha$, the numerator of $\tilde{\eta}$ is negative while the denominator is positive, so that $\tilde{\eta}$ is negative. As $\eta$ is

a value between 0 and 1 , the assertion $\eta>\tilde{\eta}$ is always true, and $\delta_{c} \delta_{c c}=\frac{c}{a}<1$.

When $\gamma>1-2 \alpha$, we have that $\gamma>\bar{\gamma}($ as $\bar{\gamma}<1-2 \alpha)$ and $\alpha>\frac{1}{3}$ (because of the assumption

$\gamma<\alpha)$. Here as $\gamma>1-2 \alpha$ we always have $\tilde{\eta}>\bar{\eta}$.

The externality plays a crucial role and is necessary to choose $\eta>\bar{\eta}$ to obtain $f(1)<0$. As seen

before, the existence of $\delta_{c}$ and $\delta_{c c}$ in $] 0,1[$ still requires $\eta>\tilde{\eta}$. However, as $\gamma>1-2 \alpha$ we always have $\tilde{\eta}>\bar{\eta}$. Therefore the conditions $\eta>\tilde{\eta}$ and $\eta<\bar{\eta}$ can never be satisfied simultaneously.

b.3) Conclusion for the case $\alpha<\frac{1}{2}$

There are three possibilities:

(i) $\delta_{c}$ and $\delta_{c c}$ smaller than 1: true if $f(1)>0$ and $\delta_{c} \delta_{c c}<1$.

(ii) $\delta_{c}<1$ and $\delta_{c c}>1$ : true if $f(1)<0$. 
(iii) $\delta_{c}$ and $\delta_{c c}$ larger than 1: true if $f(1)>0$ and $\delta_{c} \delta_{c c}>1$.

Then we obtain:

- Proof of lemma 3 (ii): When $\gamma<1-2 \alpha$ (here $0<\alpha<\frac{1}{2}$ ) we have that $f(1)>0$ and that $\delta_{c} \delta_{c c}<1$. Then there exist $\delta_{c}$ and $\delta_{c c}$ are in ] 0,1 [. Therefore, there exist an interval in ]0,1[ on which $\rho_{c}$ is negative (the first set of conditions in Lemma 3 (ii) are due to the fact that the assumption $\gamma<\alpha$ is binding for $\alpha<\frac{1}{3}$ ).

- Proof of Lemma 3(i), first set of conditions): When $\gamma>1-2 \alpha$ the externality must be chosen larger than $\bar{\eta}$ in order to have a negative solution $\rho_{c}$ in which case it exists $\bar{\delta}$ in $] 0,1[$ such that $f(\delta)>0$ on $] \bar{\delta}, 1\left[\right.$. Otherwise, $\rho_{c}$ is strictly positive. Note that $\bar{\eta}$ is smaller than 1 under the previous assumptions.

Case B: $\alpha>\frac{1}{2}$ (Proof of Lemma 3(ii), second set of conditions)

In this case, $f$ is a concave function with $f(0)>0$. This is sufficient to prove the existence of the roots $\delta_{c}$ and $\delta_{c c}$. Let $\bar{\delta}$ be the unique positive root. If $f(1)$ is negative then $\bar{\delta}$ is in ]0,1[. First, note that $\alpha$ larger than $\frac{1}{2}$ implies $2 \alpha+\gamma-1>0$. Then, from the expression for $f(1)$ given above, $f(1)<0$ for $\eta>\frac{2 \alpha+\gamma-1}{1-\gamma}=\bar{\eta}$. Finally, $\bar{\eta}$ is smaller than 1 only if $\gamma<1-\alpha$.

Q.E.D.

\subsection{Proof of Lemma 3: Identification of the roots}

Since for $\rho \rightarrow-\infty$ both eigenvalues are negative, $B$ is clearly positive. Then, the convention $\left|p_{1}\right|<\left|p_{2}\right|$ implies $p_{1}=\frac{-B+\sqrt{B^{2}-4 C}}{2}$ and $p_{2}=\frac{-B-\sqrt{B^{2}-4 C}}{2}$. From the fact that the graph of $p_{1}$ is upward sloping as $\rho \rightarrow-\infty$ (and inversely for $p_{2}$ ) it follows that

$$
\begin{gathered}
\lim _{\rho \rightarrow-\infty} p_{1}<-1 \Longleftrightarrow \lim _{\rho \rightarrow-\infty} \sqrt{B^{2}-4 C}<\lim _{\rho \rightarrow-\infty} B-2 \\
\lim _{\rho \rightarrow-\infty} p_{2}>-1 \Longleftrightarrow \lim _{\rho \rightarrow-\infty}-\sqrt{B^{2}-4 C}>\lim _{\rho \rightarrow-\infty} B-2
\end{gathered}
$$

If $\lim _{\rho \rightarrow-\infty} B-2$ is negative then $\lim _{\rho \rightarrow-\infty} p_{1}>-1$ and any solution $\rho_{c}$ is a solution to $p_{2}=-1$. Similarly, if $\lim _{\rho \rightarrow-\infty} B-2>0$ then $\lim _{\rho \rightarrow-\infty} p_{2}<-1$, and $\rho_{c}$ is a solution to $p_{1}=-1$. 
Study of $\lim _{\rho \rightarrow-\infty} B-2$

The limit can be considered as a function of $\delta$, all others parameters $(\alpha, \gamma, \eta)$ being fixed (indeed, the eigenvalues $p_{i}$ are functions of $\rho$ members of a family generated by the parameter $\delta$ ). Using the definition we obtain,

$$
\lim _{\rho \rightarrow-\infty} B-2=\frac{T_{11}}{\alpha^{2} \delta T_{12}} g(\delta)
$$

with $g(\delta)=(1-\alpha)(1-3 \alpha-\alpha \eta) \delta^{2}+\left(\alpha^{2}-\gamma(1-2 \alpha)-\eta \alpha(\alpha-\gamma)\right) \delta+\gamma^{2}$.

As $T_{11}$ and $T_{12}$ are both negative, we focus the analysis on the sign of $g(\delta)$ on ]0,1[. As we are only concerned by $\delta$ 's such that the solution $\rho_{c}$ exists, we assume that the conditions of Lemma 3 (i) are satisfied, i.e. $\delta$ in $I_{\delta 1}$.

Let $g(\delta)=a \delta^{2}+b \delta+c$, with $c$ strictly positive. Clearly, $g(\delta)$ is not strictly negative on the interval ]0,1[ because $c>0$.

Two cases need to be considered depending on whether $g$ is concave or convex. This property depends on the sign of the coefficient $a$. We have:

$$
a>0 \Longleftrightarrow \eta<\frac{1-3 \alpha}{\alpha}=\eta_{a}
$$

It is easy to see that if $\alpha<\frac{1}{4}$ then $\eta_{a}>1$ and $\eta<\eta_{a}$. Consequently, $a$ is strictly positive, $\forall \eta$, and $g$ is convex.

If $\alpha \in] \frac{1}{4}, \frac{1}{3}\left[\right.$ and $\eta<\eta_{a}$, then $a>0$.

Finally, if $\alpha>\frac{1}{3}, a<0, \forall \eta$, because $\eta_{a}$ is negative.

Consider first $\alpha>\frac{1}{3}$ together with the others conditions given in Lemma 3(i). As $g$ is concave, the sign of $g(1)$ need be analyzed. We have

$$
g(1)=(2 \alpha+\gamma-1)^{2}-\alpha(1-\gamma) \eta
$$

The sign of $g(1)$ can be expressed in term of $\eta$ :

- $g(1)>0 \Longrightarrow \eta<\tilde{\eta}$

- $g(1)<0 \Longrightarrow \eta>\tilde{\eta}$

On the other hand, the conditions of Lemma $3(i)$ imply an externality $\eta$ larger than $\bar{\eta}$. It is easy to verify that under the assumptions of Lemma $3(i)$, we have $\tilde{\eta}<\bar{\eta}$ implying $g(1)<0$.

Therefore, when $\alpha>\frac{1}{3}$ the function $g$ is negative on an interval $] \delta_{g}, 1\left[\right.$, with $\delta_{g}>0$. On the other hand, Lemma 3 (i) imply the existence of $\bar{\delta}$ such that $\rho_{c}$ is negative for all $\delta$ in ] $\bar{\delta}, 1\left[\right.$. It can be shown that $\bar{\delta}$ is larger than $\delta_{g}$. Therefore, the assumption of the existence of $\rho_{c}$ implies then $g(\delta)<0$, for all $\delta$ in $I_{\delta}$. 
To conclude, under the assumption $\alpha>\frac{1}{3}$, the eigenvalue $p_{2}$ will then be larger than -1 when $\rho$ tends to infinity. Therefore, there is indeterminacy for $\rho<\rho_{c}$.

Consider now the conditions given in Lemma 3 (ii). A detailed characterization of all the possible cases is feasible, but tedious. Instead, we give numerical examples showing that both cases can occur within the feasible range of parameters.

For $\alpha<\frac{1}{4}$, as seen before, the coefficient $a$ is positive, so $g$ is convex. Let $\alpha=0.2$ and $\gamma=0.16$. Then for $\eta=0.1$, the function $g$ is strictly positive on $I_{\delta c}$, while it is strictly negative if $\eta=0.9$. Therefore both cases can arise, but the second case needs a sufficiently high externality, which depends on $\alpha$ and $\gamma$.

For $\alpha$ between $\frac{1}{4}$ and $\frac{1}{3}$, the sign of $a$ varies with the externality. For $\alpha=0.3$ and $\gamma=0.2$, $g(\delta)>0$ if $\eta=0.1$, but $g(\delta)<0$ for $\eta=0.5$ on $I_{\delta}$.

Finally, for $\alpha>\frac{1}{3}$ and $\gamma<1-2 \alpha$, the function $g$ can also be either positive or negative: for $\alpha=0.35, \gamma=0.2$ and $\eta=0.1, g(\delta)$ is positive, while it is negative for $\eta=0.5$. Again, it is the increase in $\eta$ that shifts the graphs of $p_{1}$ and $p_{2}$ upward (from case B to case C).

Q.E.D.

\subsection{Proof of Proposition 1 (i).}

The social utility function is defined by

$$
u(x)=\operatorname{Max} \mu_{a} N_{a} u_{a}\left(x_{a}\right)+\mu_{b} u_{b} N_{b}\left(x_{b}\right)+\left(1-\mu_{a}-\mu_{b}\right) N_{c} u_{c}\left(\left(x-N_{a} x_{a}+N_{b} x_{b}\right) / N_{c}\right)
$$

The first and second order derivatives of the social utility function can be related to the derivatives of the individual utility function of the agents. Indeed, the first order conditions associated to the maximization problem that define the social utility function give

$$
\begin{aligned}
& \Psi^{1}\left(x_{a}, x_{b}, x ; \mu_{a}, \mu_{b}\right)=\mu_{a} N_{a} u_{a}^{\prime}\left(x_{a}\right)-\left(1-\mu_{a}-\mu_{b}\right) N_{a} u_{c}^{\prime}\left(\left(x-N_{a} x_{a}+N_{b} x_{b}\right) / N_{c}\right)=0 \\
& \Psi^{2}\left(x_{a}, x_{b}, x ; \mu_{a}, \mu_{b}\right)=\mu_{b} N_{b} u_{b}^{\prime}\left(x_{b}\right)-\left(1-\mu_{a}-\mu_{b}\right) N_{b} u_{c}^{\prime}\left(\left(x-N_{a} x_{a}+N_{b} x_{b}\right) / N_{c}\right)=0
\end{aligned}
$$

Then the following expressions are easily obtained

$$
\begin{aligned}
u^{\prime}(x) & =\left(1-\mu_{a}-\mu_{b}\right) u_{c}^{\prime}\left(\left(x-N_{a} x_{a}+N_{b} x_{b}\right) / N_{c}\right)=\mu_{a} N_{a} u_{a}^{\prime}\left(x_{a}\right) \\
u^{\prime \prime}(x) & =\mu_{a} N_{a} u_{a}^{\prime \prime}\left(x_{a}\right) \frac{\partial x_{a}}{\partial x}
\end{aligned}
$$

where $x$ represents the aggregate consumption. The implicit function theorem applied to $\Psi$ allows to express $x_{a}$ as a function of $x$ near the steady state $\left(x_{a}^{*}, x_{b}^{*}, x^{*}\right)$. In matrix form we can write,

$$
\left(\begin{array}{l}
\frac{\partial x_{a}}{\partial x} \\
\frac{\partial x_{b}}{\partial x}
\end{array}\right)=\left(\begin{array}{ll}
\frac{\partial \Psi^{1}}{\partial x_{a}} & \frac{\partial \Psi^{1}}{\partial x_{b}} \\
\frac{\partial \Psi^{2}}{\partial x_{a}} & \frac{\partial \Psi^{2}}{\partial x_{b}}
\end{array}\right)^{-1}\left(\begin{array}{c}
\frac{\partial \Psi^{1}}{\partial x} \\
\frac{\partial \Psi^{2}}{\partial x}
\end{array}\right)
$$


Some straightforward computations give

$$
x_{a}^{\prime}\left(x^{*}\right)=\frac{\partial x_{a}^{*}}{\partial x}=\frac{\mu_{c} \mu_{b} u_{b}^{\prime \prime}\left(x_{b}^{*}\right) u_{c}^{\prime \prime}\left(x_{c}^{*}\right)}{\mu_{a} \mu_{b} N_{c} u_{a}^{\prime \prime}\left(x_{a}^{*}\right) u_{a}^{\prime \prime}\left(x_{a}^{*}\right)+\mu_{a} \mu_{c} N_{b} u_{a}^{\prime \prime}\left(x_{a}^{*}\right) u_{c}^{\prime \prime}\left(x_{c}^{*}\right)+\mu_{b} \mu_{c} N_{a} u_{b}^{\prime \prime}\left(x_{b}^{*}\right) u_{b}^{\prime \prime}\left(x_{b}^{*}\right)}
$$

where $\mu_{c}=1-\mu_{a}-\mu_{a}$.

The result then follows from the definition of $\rho$.

Q.E.D 